\title{
CLASSIFICAÇÃO DE JOGOS ELETRÔNICOS COMO TECNOLOGIA ASSISTIVA PARA PESSOAS COM DEFICIÊNCIA VISUAL
}

\section{ELECTRONIC GAMES CLASSIFICATION AS ASSISTIVE TECHNOLOGY FOR PEOPLE WITH VISUAL DEFICIENCY}

Claudio Gonçalves Bernardoa

Cláudio Gottschalg Duque

Tomas Roberto Cotta Orlandic

Alexandre Morid

\begin{abstract}
RESUMO
Introdução: a Classificação da Informação pertence a uma das categorias essenciais da Ciência da Informação. Estudos sobre questões relacionadas à Organização da Informação são fundamentais, pois geram reflexões sobre a informação como coisa, um objeto a ser alcançado. Objetivo: propor a classificação de jogos eletrônicos como tecnologia assistiva para a pessoa com deficiência visual, para ampliar a justificativa do uso de jogos como fonte de informação e recurso de apoio à apropriação da informação em contextos de aprendizados. Metodologia: o tipo de pesquisa adotado foi a revisão de literatura, retirando a polissemia em nomes e classes de jogos eletrônicos pesquisados. Foram identificados conceitos sobre jogos eletrônicos em pesquisa bibliográfica no quadriênio 2015-2018. Foi realizada uma diferenciação do jogo desenvolvido para entretenimento e o jogo desenvolvido para ser tecnologia assistiva para pessoa com deficiência visual. Foi criada uma organização de cada estilo de jogo nos respectivos agrupamentos. Resultados: foi gerada uma classificação de jogos eletrônicos para as pessoas com deficiência visual. Além de apresentar a classificação o artigo chama a atenção para a discussão social, que é um dos papéis da Ciência da Informação. Conclusões: A proposta não pretende criar um rótulo sobre a informação mas facilitar a compreensão de informação formatada para as pessoas com deficiência visual. Por se tratar de uma linguagem documentária pode ser aproveitada como catálogo de jogos eletrônicos disponíveis ao usuário, fomentar o diálogo sobre a informação que pode ser disponibilizada a partir do uso desses artefatos e servir de

\footnotetext{
a Doutorando em Ciências da Informação na UnB - Universidade de Brasília / FCl - Faculdade de Ciências da Informação. Email: claudiogbgb@gmail.com

b Doutor em Linguística Computacional pela Universität Giessen. Doutor em Produção e Gestão da Informação no programa de pós-graduação em Ciência da Informação pela Escola de Ciência da Informação UnB. Email: klaussherzog@gmail.com

c Doutorando em Ciências da Informação na UnB - Universidade de Brasília / FCl - Faculdade de Ciências da Informação. Email: tomasroberto@gmail.com
}

d Mestre em Ciência da Informação pela Universidade de Brasília. Email: xmorihome@gmail.com
\end{abstract}


instrumento de transformação da realidade social.

Descritores: Classificação da informação. Pessoa com deficiência visual. Jogos eletrônicos. Tecnologia assistiva.

\section{INTRODUÇÃO}

Delimitar o formato da organização e recuperação da informação é uma das atividades estudadas pelos pesquisadores em fundamentos da organização da informação. Estudar a sua natureza, quais são as necessidades apresentadas pelos usuários, bem como analisar o contexto no qual estão inseridos os usuários para compreendê-lo de maneira mais ampla, são objetos do mesmo estudo.

O entendimento das demandas por informação exige que o pesquisador compreenda as implicações, o método de transmissão e finalize identificando como a informação será interpretada, posto que não basta que exista, mas que seja interpretada no contexto global, considerando o máximo de variáveis possíveis.

A interpretação da informação depende do entendimento do trio emissormensagem-receptor e do seu veículo de disseminação. A compreensão do que se necessita como informação sem, porém, analisar como ela será disponibilizada, qual o meio de emissão, qual o meio de recepção e principalmente 0 meio de transporte poderá oferecê-la fragmentada, subdimensionada, interpretada não em sua totalidade.

Ottonicar, Santos e Moraes (2017) observam que com a aceleração da tecnologia da informação e comunicação existe uma percepção da necessidade de gerenciamento da informação para facilitar o acesso, sua organização e tratamento, porém sem perder de vista que precisa dar autonomia à pessoa receptora, respeitar seu individualismo e seus sentimentos, fomentar a sensação de empoderamento quando esta busca a informação.

Este artigo aborda a questão do jogo eletrônico que proporciona informação à pessoa com deficiência visual (PDV). O gerenciamento e a organização da informação fornecida pelos artefatos eletrônicos à PDV permitem 
Claudio Gonçalves Bernardo, Cláudio Gottschalg Duque, Tomas Roberto Cotta Orlandi, Alexandre Mori Correio

Classificação de jogos eletrônicos como tecnologia assistiva para pessoas com deficiência visual

ampliar a visão de inclusão social, principalmente quando os jogos servem como Tecnologia Assistiva (TA).

A presente pesquisa aborda o problema da falta de classificação de jogos eletrônicos, como tecnologia assistiva e não como entretenimento para a PDV, posto que não existe hoje uma classificação reconhecida de jogos eletrônicos empregados como tecnologia assistiva.

A classificação dos jogos eletrônicos que servem de TA para a PDV é um pequeno passo para a organização do conhecimento sobre o que thes é ofertado como produto de consumo. A intenção é classificar para facilitar a compreensão das ideias, do conhecimento, posto ser de grande importância. Esta visão é bem apropriada no texto de Moreira e Moraes (2019):

[....] esse nível de importância pode ser atribuído ao fato de ser classificação uma atividade natural e indispensável à relação do homem com o mundo, tanto no que se refere à organização de objetos quanto à organização de ideias ou de conhecimento. Nesse nível de classificação visa-se classificar as coisas com o fim de simplificar (no sentido de tornar compreensível) o mundo e dotá-lo de sentido, o que é realizado por meio do agrupamento de coisas semelhantes numa estrutura que revela o modo como a realidade é ou pode ser compreendida (MOREIRA; MORAES, 2019, p. 227).

Para atingir o objeto desta pesquisa foi necessário abordar a classificação no contexto da Ciência da Informação $(\mathrm{Cl})$, pesquisar todos os jogos disponibilizados na literatura, identificar quais são desenvolvidos para a pessoa vidente e quais são desenvolvidos para a PDV. Dentre esses últimos é necessário ainda identificar quais foram desenvolvidos para servir apenas de entretenimento e quais foram desenvolvidos para servir como TA, já que esses servem de recursos de apoio à apropriação da informação em contextos de aprendizados.

A pesquisa apresentada em (BERNARDO et al, 2016) é uma das motivadoras do presente artigo, posto que se trata de proposta de diretrizes para jogos eletrônicos em ambientes assistivos para a PDV, levando em conta que nos recentes anos tem aumentado o interesse na aplicação de jogos eletrônicos para determinados contextos, tais como cuidado com saúde, reabilitação motora e mental, esportes e terapias ocupacionais. 


\section{CIÊNCIA DA INFORMAÇÃO}

Por ser uma ciência interdisciplinar e de acordo com (BUCKLAND, 2018) se quisermos falar sobre disciplinas devemos perceber que a Ciência da Informação é uma "superdisciplina" baseada em todas as outras, por isso a mesma deve ser entendida sob um ponto de vista global, em uma visão macroscópica mas principalmente sob uma visão específica, detalhada em uma visão microscópica. Considerando essa visão mais apurada sob seus detalhes é de primordial importância existir uma linguagem documentária, como a classificação. Uma das propostas da classificação é facilitar a recuperação da informação em qualquer sistema, sendo a única forma de organizar um número grande de objetos relacionados.

Buckland (2018) defende que o objetivo do cientista da informação é capacitar todos a se tornarem mais informados e menos ignorantes e para isso é primordial fazer uso de todas as técnicas e ideias que possam ser úteis, incluindo artefatos digitais. Não existe argumento que faça crer que a PDV não esteja nesse grupo de pessoas, mesmo com as particularidades que apresenta.

Segundo Sousa e Araújo Junior (2017) a organização da informação ocorre em um sistema que consiste de componentes inter-relacionados para coletar, processar e disseminar informações, sob uma gestão de um encarregado, aparecendo com um mecanismo de feedback para auxiliar na revisão, melhoria e adaptação ao ambiente em que atua. Afirmam que por ser uma linguagem documentária e atender demandas da gestão da informação uma classificação apresenta a característica de começar no geral e ir para o específico, particularizado, na qual se encaixa o público alvo desta pesquisa. Observam ainda que é relevante perceber que ela, a informação é perene e não datada, por isso não cai em obsolescência.

Uma classificação deve ser reconhecida como uma linguagem. Dahlberg (1978) conceituou:

Podemos dizer que a linguagem constitui a capacidade do homem designar os objetos que o circundam assim como de comunicar-se com os seus semelhantes. As linguagens utilizadas nas necessidades da vida diária denominam-se 
linguagens naturais. Além destas o homem criou outras, chamadas linguagens especiais ou linguagens artificiais ou linguagens formalizadas, como a linguagem da química, linguagem da matemática, linguagem da lógica, linguagem dos sistemas de classificação, etc. (DAHLBERG, 1978).

A Organização do Conhecimento, segundo Dahlberg (2006), é a ciência que sistematicamente estrutura e organiza unidades de conhecimento de acordo com as características de seus elementos e a aplicação desses conceitos a objetos e sujeitos. Dahlberg (2006) define duas aplicações para organização do conhecimento: "a) a construção de sistemas conceituais; b) a correlação de unidades desse sistema conceitual com objetos da realidade".

Alguns princípios básicos de classificação são adotados na Taxonomia. Sousa e Araújo Júnior (2013) declaram que esta pode ser definida como um sistema de classificação que apoia o acesso à informação, permitindo classificar, alocar, recuperar e comunicar informações em um sistema de maneira lógica. Esses autores a apresentam como um instrumento para a classificação de documentos, onde tal vinculação tem a finalidade de estruturar, sistematizar e padronizar o conjunto de assuntos correspondentes às atividades desempenhadas pelas áreas de trabalho de uma organização, garantindo aos tomadores de decisão o acesso mais rápido aos documentos e informações para processo decisório.

Segundo Robredo e Bräscher (2010) no domínio das representações do conhecimento as taxonomias são instrumentos que organizam logicamente os conteúdos informacionais. O conceito de organização remete para um procedimento classificatório e permite agrupamento categorizado, isto é, a partir de um assunto formam-se categorias que se dividem em classes e subclasses hierarquicamente, formando uma lista de categorias de assunto estruturada, reforçando o papel das linguagens documentárias para a organização e a recuperação da informação

Antes aplicada à Biologia e à Lógica, a Taxonomia passou a ser usada no campo da tecnologia e no contexto da Ciência da Informação, para servir aos sistemas de classificação (CURRÁS, 2010). Por ser um estudo científico que classifica de uma forma sistemática elementos e os acondiciona em categorias, 
os elementos em que forem reconhecidas afinidades entre si estarão na mesma categoria, aqueles que não tiverem afinidades serão classificados em outra categoria, e isto dá importância ao reconhecimento das afinidades entre os elementos.

É importante criar uma classificação a partir da proximidade com os usuários da informação, identificando as características e reconhecendo as categorias em que cada elemento deverá ser acondicionado.

Por estar intrinsecamente ligada à busca e à recuperação da informação, cada classificação criada deverá ter uma regra definida, onde os nomes a serem criados possam permitir uma identificação correta, sem polissemia, para evitar dubiedades por serem termos com sentido unívoco. Nessa característica está a (quase) garantia que a busca e a recuperação da informação serão potencializadas.

Diz-se quase, por sabermos que nada é por definição definitivo, nada no campo da Ciência da Informação é plenamente encerrado e, mesmo que haja consenso entre os pesquisadores é primordial reconhecer que por ser uma ciência humana, este consenso de hoje poderá ser o dissenso do amanhã. Ela deve permitir que se reflita com clareza o objetivo a que se deseja.

A construção de uma proposta de classificação não pretende criar um rótulo sobre determinada informação, mas facilitar o caminho da compreensão da informação que se quer construir e por consequência, recuperar. De acordo com Cunha e Araújo Junior (2017) o registro do conhecimento deve trazer uma série de benefícios na conversão de conhecimento tácito para explícito, e entre estes benefícios podemos destacar aqueles que poderão se valer das potencialidades das classificações na descrição padronizada de métodos e processos organizacionais: identificação do conhecimento: competências e áreas de conhecimento que as sustentam; aquisição de conhecimento: fontes de informação; seleção e validação do conhecimento: valor da informação; organização e armazenamento: acesso à informação; uso do conhecimento no cotidiano organizacional ou para processos de inovação e criação de novos conhecimentos. 
Para a classificação apresentada neste artigo foi necessário considerar a percepção da relevância da informação apontada por Monteiro et al. (2017), que afirmam que por estar associada ao significado o problema reside em informar o que é relevante para o ser humano, pois até mesmo entre as pessoas há divergências em determinar com unanimidade o que é relevante e o que não é, já que é uma questão subjetiva e pessoal.

\section{JOGOS ELETRÔNICOS E A PESSOA COM DEFICIÊNCIA VISUAL}

O termo jogo digital é um termo genérico que se refere a jogos eletrônicos desenvolvidos para serem jogados em um computador, em console ou outro dispositivo tecnológico (PIVEC; KEARNEY, 2007), desta forma pode ser definido como jogo onde existe interação entre humano e computador, recorrendo ao uso de tecnologia (GEE, 2003). Para utilizar apenas um para este artigo foi escolhido o termo jogo eletrônico e alguns momentos é feito referência ao termo em inglês games.

O Brasil é o 11ํㅡㄹ país no ranking de maior mercado de jogos eletrônicos no mundo. O setor movimenta 900 milhões de reais no país a cada ano (FLEURY; NAKANO; CORDEIRO, 2014). Com o crescimento dos jogos eletrônicos muitos gêneros se estabeleceram no mercado. Sakuda e Fortim (2018) organizaram a segunda versão do censo da Indústria Brasileira de Jogos Digitais (IBJD), elaborado por uma equipe multidisciplinar, no âmbito do acordo de cooperação técnica firmado entre o Ministério da Cultura, a Agência Brasileira de Cooperação e a Organização das Nações Unidas para a Educação, a Ciência e a Cultura (UNESCO). A pesquisa teve por objetivo informar sobre a evolução do setor de games no Brasil, atualizando dados estatísticos e facilitando o desenho de políticas para o seu desenvolvimento.

No desenvolvimento desse jogo compreende-se que a intenção do desenvolvedor é que seu produto seja entendido pelo usuário final. Daí é possível definir para a classificação dos jogos eletrônicos, inserida no contexto de classificação da informação o mesmo que Cunha e Araújo Junior (2017) definem quando afirmam que esta é funcional uma vez que orienta a análise de 
distorções pela segregação, excluindo redundâncias, erros e distorções identificados da linguagem natural ao fazer uso de uma estrutura de classificação padronizada em linguagem documentária.

Muitos desses jogos são desenvolvidos a partir de requisitos bem elaborados, com foco no público-alvo. Infelizmente não são todos e para a PDV esse quadro se agrava mais porque não existe padronização dos conceitos relacionados aos jogos eletrônicos. Pensar no jogo como vetor informacional, como instrumento de fonte de informação para a PDV é diferente de oferecer apenas uma proposta da jogabilidade.

Segundo Sakuda e Fortim (2018) as atividades que compõem as principais faces do design de jogos eletrônicas são: game design, programação, arte, design, gestão de projetos, roteiro, animação, pesquisa, controle de qualidade, sonorização, produção executiva, propapganda e publicidade, monetização, consultoria, serviços educacionais, inteligência de negócios, localização, treinamento corporativo, outras atividades digitais e tecnológicas, outras atividades culturais e criativas, outras atividades.

Uma alternativa atual para os jogos para PDV são jogos de áudio, aqueles que não são necessariamente destinados a pessoas com deficiência visual (FLEURY; NAKANO; CORDEIRO, 2017). Estes são mais simples em comparação com os principais do mercado e proporcionam ao jogador uma experiência multiplayer, diversidade e longevidade igual aos principais títulos de jogos. Além de uma forma de entretenimento, os audiogames promovem o desenvolvimento da audição, aperfeiçoam a capacidade de percepção do ambiente apenas pelo som e ainda ajudam a aumentar a confiança e autoestima (SANTOS, 2016).

Muriel e Crawford (2018) defendem que certas áreas da realidade social estão sendo gamificadas, isto é, estão usando elementos de jogos e aplicandoos a outras áreas como educação, trabalho, terapia, negócios, guerra, desenvolvimento social e relacionamentos, entre outros. Para esses pesquisadores a realidade social é transformada em um jogo eletrônico e ao fazê-lo, a cultura dos jogos eletrônicos afeta significativamente a sociedade 
como um todo. Portanto, embora possa haver atores sociais que os ignore como cultura, a cultura dos jogos os está afetando e a todos nós.

Orlandi et al. (2018) apresentam o conceito de gamificação na educação abordando 0 aspecto motivacional dos alunos afirmando fazer-se necessário 0 uso de estratégias para motivá-los na jornada de aprendizagem. O uso de vídeos, podcasts e blogs capturam o interesse dos alunos e já foram plenamente discutidos, mas os usos de jogos eletrônicos são apresentados como mais uma estratégia, por ser instrumento que traz informação e pode ser moldado para qualquer público incluindo pessoas com deficiência visual. O jogo eletrônico em alguns contextos serve de instrumento de apropriação da informação para os seus usuários alterando a sua realidade.

Existem vários gêneros de jogos eletrônicos. Segundo Costa (2014) são:

i. Jogos de ação: Este gênero exige que o jogador tenha uma boa coordenação entre mãos e olhos, tendo ênfase na reação instantânea. As respostas rápidas do jogo são importantes neste gênero.

ii. Jogos de Simulação: neste gênero o objetivo é reproduzir com fidelidade um fenômeno ou acontecimento real. Por simularem situações reais, existem várias possibilidades para desenvolvedores desse gênero, principalmente com os avanços computacionais atuais, onde físicas realistas podem ser aplicadas para uma maior aproximação da situação real a ser simulada.

iii. Jogos de aventura: Estes jogos começaram baseados em texto, antes chamados de text-based games. Neste estilo o jogador é inserido em um mundo onde necessita gerenciar recursos, resolver problemas e desafios apresentados. Precisa não somente agir, mas pensar nas consequências de suas ações e analisar a situação como um todo, um pouco diferente dos jogos de ação onde agir por instinto é necessário.

iv. Jogos de estratégias: Jogos de estratégia podem acontecer em ambientes fictícios ou históricos, seu principal foco são pensamento e planejamento. Os jogos de estratégia em tempo real (Real Time Strategy - RTS) são o subgênero dominante dos jogos de estratégia, tendo um ritmo acelerado e 
são baseados nos "quatro x", que significam expansão, exploração, extração e extermínio.

v. Turn based. Nestes o jogador conta com um ritmo de jogo mais lento, com tempo para poder montar sua estratégia.

vi. Jogos de esportes. São baseados em competições esportivas e esportes. Depende da habilidade do jogador, exigindo que o jogador tenha uma boa noção do que está acontecendo na tela. Existe um subgênero que é o de gerenciamento de esportes, onde o jogador gerencia clubes e equipes, podendo ser de esportes variados também. Nesse subgênero o jogador é mais exigido na parte do raciocínio, tendo uma jogabilidade mais tranquila.

Os jogos eletrônicos podem atuar em muitas áreas tais como aprendizagem, desenvolvimento lógico e motor, interação social e relação com culturas diferentes. Com a tecnologia cada vez mais presente na saúde, comunicação, educação e várias outras áreas, estes jogos foram inseridos em diversos meios, demonstrando utilidades além da diversão, como as propostas de jogos em ambiente virtual desenvolvidos para a reabilitação das funções executivas e do desempenho funcional de pessoas com esquizofrenia, baseadas em tarefas simples das atividades instrumentais da vida diária (IGNÁCIO, 2016).

Esses jogos eletrônicos servem para a PDV como produtos para Tecnologia Assistiva. O trabalho de Matsuhara e Fernandes (2017) apresenta uma lista atualizada de produtos assim desenvolvidos para as PDV, dentre eles muitos ampliadores e leitores de tela, elementos de leitura digital, recursos para acessibilidade visual em aparelhos mobile e dispositivos para mobilidade. As autoras discutem o alcance ao maior número de informações que uma pessoa pode obter, permitindo a ela a inclusão social, econômica e cultural com melhor qualidade de vida.

\subsection{Jogos Educativos}

Os jogos também têm sido utilizados na área da educação, proporcionando uma melhor experiência de aprendizado devido à sua 
abordagem leve e descontraída. Para poder ser utilizado como instrumento educacional principalmente para as pessoas com deficiência visual os jogos eletrônicos devem conter algumas características específicas para atender as necessidades vinculadas à aprendizagem. Segundo Pietro (2005, apud SAVI; ULBRICHT, 2008) os jogos educacionais devem possuir objetivos pedagógicos e sua utilização deve estar inserida em contexto e situação de ensino baseados em metodologia que oriente o processo, através da interação, da motivação e da descoberta, facilitando a aprendizagem de um conteúdo.

Com o objetivo de caracterizar o jogo como fonte de informação e instrumento para a apropriação da informação pela PDV é fundamental empoderar o discurso de muitos professores quando defendem que os jogos além de facilitarem a aquisição de conteúdo, contribuem também para o desenvolvimento de uma grande variedade de estratégias que são importantes para a aprendizagem, como resolução de problemas, raciocínio dedutivo e memorização (McFARLANE; SPARROWHAWK; HEALD, 2002 apud SAVI; ULBRICHT, 2008). Outros benefícios dos jogos e simuladores incluem a melhoria do pensamento estratégico e insight, melhoria das habilidades psicomotoras, desenvolvimento de habilidades analíticas e habilidades computacionais (MITCHELL; SAVILL-SMITH, 2004 apud SAVI; ULBRICHT, 2008). Alguns jogos online, que são disputados em equipes, ajudam a aprimorar o desenvolvimento de estratégias em grupo e a prática do trabalho cooperativo (GROS, 2003 apud SAVI; ULBRICHT, 2008).

\subsection{E-SPORTS E O MERCADO dE Jogos Eletrônicos}

Um grande exemplo da variação do mercado de jogos eletrônicos é o fenômeno dos esportes eletrônicos ou e-sports, que vêm crescendo mundialmente com campeonatos mundiais de vários jogos, com grandes premiações em dinheiro, bem organizados e que atraem uma enorme quantidade de jogadores e admiradores.

No Brasil este cenário dos esportes eletrônicos está em crescimento, sendo uma pequena fatia importante quando comparada com o cenário mundial. 
O mercado de games no Brasil movimenta atualmente $\mathrm{R} \$ 1$ bilhão e estima que movimente US $\$ 696$ milhões ( $R$ \$ 2,15 bilhões) e audiência de 385 milhões de pessoas em todo o mundo, com a maior concentração nos EUA e Ásia. O jogo que mais arrecadou foi o mundialmente conhecido League of Legends, ou LoL para os íntimos. No mundo, estima-se mais de 100 milhões de gamers, profissionais ou não, e o Brasil está em quarto lugar no número de participantes (FURTADO, 2017).

Nos últimos anos o mercado de jogos eletrônicos em geral vem crescendo cada vez mais. Gonçalves (2016) afirma que a indústria de games faturou no mundo todo US\$ 91 bilhões ao longo de 2016. Os jogos mobile foram responsáveis pela maior parte da arrecadação, cerca de US\$ 41 bilhões, impulsionados especialmente pelos sucessos "Pokémon Go" e "Clash Royale". Nos PCs, "League of Legends" ficou com o posto de jogo mais rentável no ano, lucrando para sua produtora, a Riot Games, cerca de US\$150 milhões por mês. "Dota 2" ficou com a segunda posição, rendendo US\$23,4 milhões mensalmente. Afirma ainda que o mercado está prestes a experimentar novas plataformas de mídia que podem expandir seu alcance.

\subsection{Jogos Eletrônicos para Pessoa com Deficiência Visual}

Nos jogos eletrônicos para pessoas com deficiência visual foi verificado que a parte de áudio dos jogos tem muito mais importância. Em alguns casos é a única característica perceptível pelo jogador. Embora seja um aspecto de imersão importante, o desenvolvimento de áudio nos jogos não recebe a mesma atenção do projeto visual (PATIKOWSKI, 2013). Frequentemente, o feedback sonoro fornecido pelos jogos não é suficiente para indicar todas as informações essenciais para entendimento de um cenário, orientação em um ambiente ou navegação em um menu de jogo. Ainda que apoiados por Tecnologia Assistiva os jogadores PDV experimentam uma incompatibilidade generalizada de leitores de tela com os jogos, dado que esses softwares necessitam da possibilidade de interação usando apenas o teclado e das descrições textuais ocultas em cada elemento interativo. E, mesmo jogadores com baixa visão carecem de opções 
que ampliem o tamanho ou aumentem o contraste dos elementos do jogo (PATIKOWSKI, 2013).

Como a indústria de jogos eletrônicos foi se tornando cada vez mais gráfica devido ao avanço tecnológico, algumas funcionalidades foram adotadas por jogadores PDV para que os mesmos pudessem jogar. Algumas dessas opções foram criadas para eles, outras já existiam e foram adotadas, como os jogos baseados em texto. Esses incluem os jogos de aventuras de texto, jogos de tabuleiros digitais como Batalha Naval e os Multi-User Dungeons (MUD) que são jogos baseados em HTML, onde vários jogadores se conectam e jogam através de texto. Infelizmente para esse tipo de jogo o mercado distribuidor não oferece a mesma variedade de jogos que oferece para os títulos mais vendidos por serem jogos mais simples (GAME ACCESSIBILITY GUIDELINES, 2017).

Quanto aos jogos populares, alguns são jogados por pessoas visualmente limitadas utilizando mecanismos de resposta auditiva. Muitos exemplos incluem jogos de luta como Tekken, Mortal Kombat e Soul Calibur. Os jogos da franquia Grand Theft Auto são conhecidos por serem divertidos de serem jogados por PDV fazendo uso de uma ferramenta de som avançada, porém são poucos os jogos populares que proporcionam uma resposta sonora avançada dessa forma. E mesmo que alguns desses jogos sejam jogados por PDV, não são completamente acessíveis por contarem com menus visuais sem nenhuma resposta auditiva (GAME ACCESSIBILITY GUIDELINES, 2017).

Os jogos de áudio são os que consistem de som e têm somente áudio, sem nenhuma parte gráfica. Estes não são especificamente jogos para a comunidade PDV, mas desde que não se precisa de visão para poder jogá-los, muitos são desenvolvidos para e por esta comunidade. Há mais de 100 jogos de áudio, incluindo jogos do gênero corrida, terror, aventura, guerra, etc. Esse tipo de jogo é desenvolvido por pequenas companhias dedicadas à área, desenvolvedores de jogos amadores, pesquisadores acadêmicos em projetos de pesquisa e pelos próprios jogadores. A maioria é bem simples se comparada aos jogos eletrônicos do mercado principal de jogos e não são encontrados com a 
mesma variedade (GAME ACCESSIBILITY GUIDELINES, 2017).

$O$ projeto desenvolvido pela organização grega SciFY (2018) que desenvolve os jogos Listen and Play (LEAP) ajuda crianças cegas para que se tornem familiarizadas com computadores, recebendo novas habilidades com diversão e aprendizado. Seu primeiro jogo chamado Tic Tac Toe ensina as crianças a usar chaves básicas do computador e ensina como se mover para um espaço de som bidimensional; no jogo chamado Tênis, as crianças sentem o movimento e a velocidade do som por meio de três dimensões e interagem nela utilizando o processamento binaural, entre outros. Esta organização mantém o site www.gamesfortheblind.org (2018) de onde é possível fazer download nos sistemas operacionais Windows, Mac OS X e Linux. A organização também mantém o projeto Memor-i com download`s de seus jogos desenvolvidos para crianças cegas (MEMORI-I, 2018).

Existem jogos que são acessíveis por meio de design original. Há alguns exemplos de jogos eletrônicos cuja acessibilidade para pessoas com deficiência visual tem sido feita por meio do design do jogo. Infelizmente, não há muitos exemplos desse tipo de jogo, não havendo também grande variedade como possui a indústria de jogos principais. Alguns jogos são frequentemente modificados pelos próprios jogadores para adicionarem funcionalidades diferentes. Portanto, por essas modificações é possível adicionar funcionalidades de acessibilidade, permitindo que jogadores com deficiência visual joguem os que foram desenvolvidos para normovisuais. Apenas um exemplo desse tipo de jogo é conhecido como Accessible Quake e foi realizada modificação tornando-o acessível para as PDV, desenvolvida pela empresa AGRIP (GAME ACCESSIBILITY GUIDELINES, 2017).

Novas tecnologias para a melhoria da experiência dos jogadores videntes são sempre desenvolvidas, porém, segundo Sens e Pereira (2015) observam que não é observada essa mesma preocupação, interesse ou esforço com pessoas que apresentam alguma deficiência. Alguns fabricantes reforçam a importância da acessibilidade, inclusive sob o ponto de vista mercadológico, contudo na prática, essa prática inclusiva não se verifica plenamente (SENS; 
PEREIRA, 2015).

Para observar se o mercado principal de jogos procura prover acessibilidade a jogadores com deficiência visual, o quadro 1 apresenta uma pesquisa dos 10 jogos mais vendidos de todos os tempos até os dias atuais, publicada por VGChartz (2017), que hospeda uma base de dados de jogos com mais de 40.000 títulos listados.

Uma pesquisa foi realizada à procura de versões acessíveis desses jogos. Dos 10 jogos da lista, apenas um foi encontrado com uma versão baseada apenas em áudio, sendo que a mesma foi desenvolvida no ano de 2014 pelo Copenhagen Institute of Interaction Design (INTERACTION AWARDS, 2014). O jogo encontrado foi o Duck Hunt do NES, de 1984, que ocupa a 10ª posição dos jogos mais vendidos até hoje. Com isso é possível perceber que uma grande parte das pessoas com deficiência visual não teve acesso a esses jogos, mesmo que estejam entre os 10 jogos mais vendidos. Isso mostra que, mesmo que tenham acesso a alguns tipos de jogos, a pessoa com deficiência visual não participa do mercado, sendo excluído do universo dos jogos eletrônicos mais populares.

Quanto a jogos eletrônicos desenvolvidos para pessoas com deficiência visual no Brasil, alguns foram criados para o sistema operacional Dosvox (2018), destinado a atender pessoas com deficiência visual. Estão disponíveis vinte e oito jogos divididos em cinco categorias: jogos educativos, jogos de RPG, passatempos, desafios e oráculos, sendo que três desses não estão dentro dessas categorias citadas, que são o jogo da forca, jogo da memorização das letras e o jogo de mistura de sons (SILVA, 2017).

Quadro 1 - Lista dos 10 melhores jogos de vendedores

\begin{tabular}{|c|c|c|c|c|}
\hline \multicolumn{6}{|c|}{ Melhores jogos de vendedores em todos os consoles de jogos } \\
\hline \multirow{2}{*}{ Ord } & Jogo & Console & Ano & $\begin{array}{c}\text { Vendas } \\
\text { (MM) }\end{array}$ \\
\hline $1^{\underline{0}}$ & Wii Sports & Wii & 2006 & 82.6 \\
\hline $2^{\underline{0}}$ & Super Mario Bros & NES & 1985 & 40.2 \\
\hline $3^{\mathbf{o}}$ & Mario Kart Wii & Wii & 2008 & 35.7 \\
\hline $4^{\mathbf{0}}$ & Wii Sports Resort & Wii & 2009 & 32.9 \\
\hline $5^{\mathbf{o}}$ & Pokémon Red/Green/Blue Version & Game boy & 1996 & 31.4 \\
\hline
\end{tabular}


Claudio Gonçalves Bernardo, Cláudio Gottschalg Duque, Tomas Roberto Cotta Orlandi, Alexandre Mori Correio

Classificação de jogos eletrônicos como tecnologia assistiva para pessoas com deficiência visual

\begin{tabular}{|c|c|c|c|c|}
\hline $\mathbf{6}^{\mathbf{0}}$ & Tetris & Game boy & 1989 & 30.3 \\
\hline $\mathbf{7}^{\mathbf{0}}$ & New Super Mario Bros & DS & 2006 & 29.9 \\
\hline $\mathbf{8}^{\mathbf{0}}$ & Wii Play & Wii & 2009 & 28.9 \\
\hline $\mathbf{9}^{\mathbf{0}}$ & New Super Mario Bros. Wii & Wii & 2009 & 28.5 \\
\hline $10^{\mathbf{0}}$ & Duck Hunt & NES & 1984 & 28.3 \\
\hline
\end{tabular}

Fonte: VGCHARTZ (2017)

\subsection{Tecnologia Assistiva e Seu papel na Ciência da Informação}

Tecnologia Assistiva (TA) significa quaisquer produtos, dispositivos, ferramentas, técnicas ou processos que ajudem uma pessoa que necessite de assistência ou esteja em fase de reabilitação, fazendo com que sua vida tenha qualidade com essa ajuda, essa facilidade. O Ministério da Educação afirma que TA é uma área de conhecimento que engloba recursos e serviços para prover ou ampliar habilidades funcionais de uma pessoa com deficiência. Seu objetivo é promover a qualidade de vida e a inclusão social de seus membros (BRASIL, 2006). Na educação, os jogos são usados para proporcionar uma melhor experiência de aprendizado devido à sua abordagem leve e descontraída. Eles contêm recursos específicos para atender às necessidades relacionadas ao aprendizado.

Meeks e Jain (2016) descrevem a TA como "dispositivos tecnológicos e software usados para tornar os materiais do curso, instrução e interações com ambientes mais acessíveis aos alunos com deficiências. $O$ objetivo da TA é remover barreiras ao ambiente educacional. Para um aluno com deficiência visual, isso pode incluir o uso da tecnologia de leitor de tela para garantir que 0 aluno possa acessar o material escrito. Ela pode ainda beneficiar estudantes sem deficiências, melhorando o acesso ao conteúdo educacional. Por exemplo, um aluno com um estilo de aprendizagem auditivo forte pode se beneficiar da capacidade de gravar palestras para reprodução em um momento posterior".

De acordo com Sartoretto e Bersch (2016), TA é até um novo termo usado no Brasil para identificar todo o arsenal de recursos e serviços que contribuem para fornecer ou expandir habilidades funcionais de pessoas com deficiência e, consequentemente, promover sua vida e inclusão independentes. Seus objetivos 
são fornecer à pessoa com deficiência maior independência, qualidade de vida e inclusão social através da expansão de sua comunicação, mobilidade, controle ambiental, habilidades de aprendizagem, trabalho e integração com família, amigos e sociedade.

Como um dos poucos estudos de usuário da informação PDV, a pesquisa de Bastos (2017) analisa os desafios encontrados no acesso à informação digital por PDV e apresenta uma vasta lista de programas e dispositivos de tecnologia assistiva em dois grandes grupos: aqueles de interfaces para usuários com baixa visão e aqueles de interfaces para usuários cegos.

Santos et al. (2018) afirmam que embora a demanda por TA no Brasil seja grande, observa-se que a produção nacional tecnológica para a pessoa com deficiência visual é insatisfatória, mesmo sendo a deficiência mais predominante na população brasileira. $\mathrm{O}$ uso dessa tecnologia possibilita a execução de atividades diárias como leitura, locomoção, comunicação e lazer, melhorando a qualidade de vida e inclusão social de seus usuários.

A produção nacional de TA é reduzida e pode ser percebido na tabela 1 que a acessibilidade ao computador (e também ao celular e ao tablet) é a menor categoria presente no Brasil, de um total de nove definidas pelo Instituto Nacional de Propriedade Intelectual - INPI (SANTOS et al., 2018). O uso de TA como vetor de informação pode ser ampliado, mesmo que seja para públicos diferentes da pessoa com deficiência visual.

Tabela 1 - Categorias de tecnologias assistivas brasileiras

\begin{tabular}{lc}
\hline Funcionalidade / Objetivo & $\%$ \\
\hline Acessibilidade ao computador & 3,7 \\
Controle do ambiente & 3,7 \\
Auxílios para vida diária & 5,6 \\
Projetos arquitetônicos & 9,3 \\
Esporte e lazer & 11,1 \\
Auxilio pessoas cegas ou com baixa visão & 14,8 \\
Educação & 14,8 \\
Mobilidade & 18,5 \\
\hline
\end{tabular}


Claudio Gonçalves Bernardo, Cláudio Gottschalg Duque, Tomas Roberto Cotta Orlandi,

\begin{tabular}{lr}
\hline Serviços & 18,5 \\
TOTAL & $\mathbf{1 0 0 , 0}$ \\
\hline
\end{tabular}

Fonte: Adaptado de Santos et al (2018)

\section{REFERENCIAL METODOLÓGICO NA CLASSIFICAÇÃO DE JOGOS ELETRÔNICOS}

Ao construir uma classificação sobre jogos eletrônicos foi identificado que recentemente pouco tem sido pensado na sua estrutura conceitual. Foram identificados dois tipos de classificação, a primeira definida em (LUCCHESE; RIBEIRO, 2009) que apresenta uma conceituação de jogos eletrônicos construída à luz da Teoria de Jogos. Nesta publicação são apresentados os principais aspectos da teoria considerados relevantes para os jogos digitas e são analisados alguns dos principais conceitos dela relacionados aos jogos eletrônicos.

A segunda classificação é aquela referente à preocupação de segmentar por faixa etária os consumidores dos jogos eletrônicos, principalmente para evitar que adolescentes ou crianças sejam apresentados a conteúdos que não condizem com a sua idade. O sistema de classificação de conteúdo de jogos eletrônicos é usado para a classificação de jogos eletrônicos em grupos relacionados com conveniência e podem ser usados como base de leis destinadas para controle de venda de jogos eletrônicos a menores.

Um primeiro passo para uma classificação é evitar que haja polissemia, nomes repetidos, mesmo que os nomes diferentes sejam parte de membros superiores hierarquicamente e diferentes. Por exemplo, na classificação apresentada na figura 1 é necessário especificar para que haja um diferenciador entre 'luta' e 'luta como esporte'. 
Figura 1 - Classificação de-para
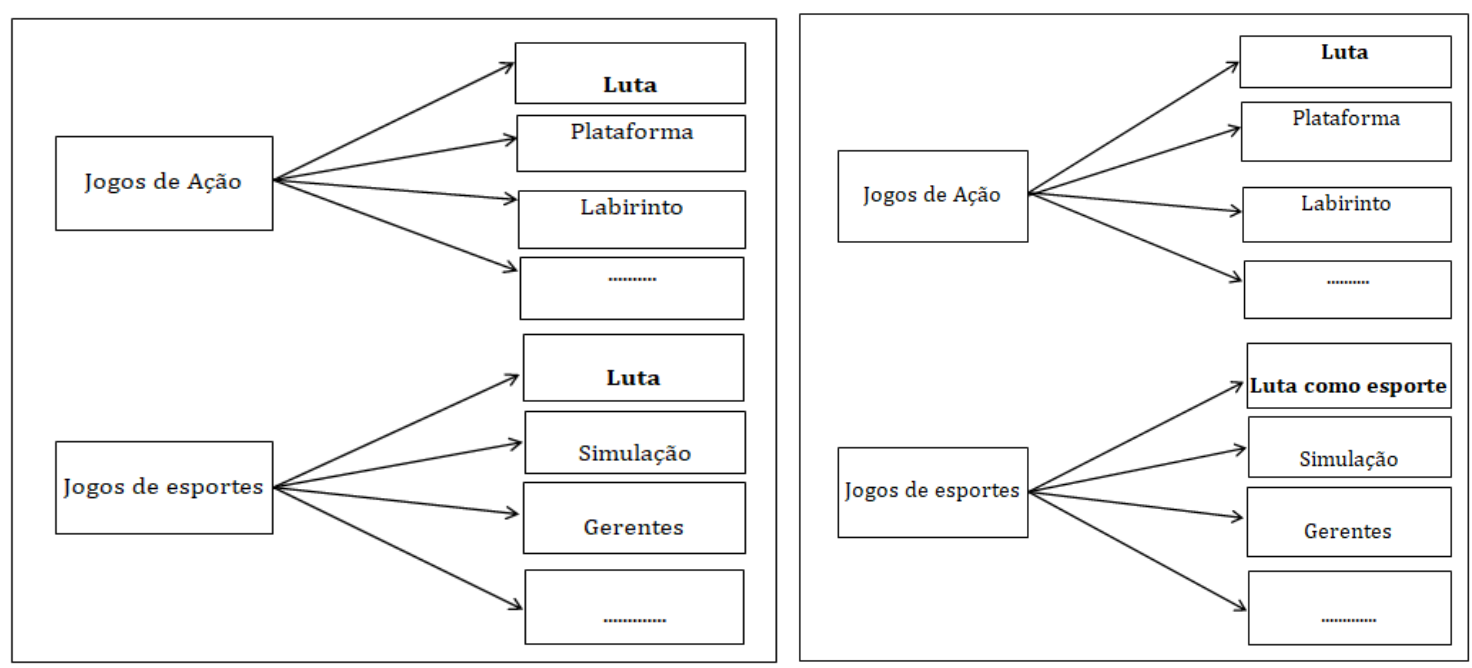

Fonte: Elaborado pelos autores (2019)

Para este artigo foi considerado o fator de publicação sobre o tema nos anos 2015 a 2018 sendo que a primeira consulta foi feita nos principais motores de busca, nacionais e internacionais. A pesquisa realizada em Scholar Google (2018), Search Yahoo (2018) e Bing (2018) imputando como filtro os argumentos de busca "eletronic games taxonomy"; "digital games taxonomy"; "taxonomia de jogos eletrônicos" e "taxonomia de jogos digitais" a partir do ano de 2015. Depois foi substituído "taxonomy" e "taxonomia" por "classification" e "classificação" respectivamente. Foram obtidas poucas publicações sendo algumas delas acadêmicas como (HUSSAIN; RASOOL, 2018) quando a maioria que fala de classificação na verdade é voltada para classificação indicativa de idade e público, que é permitido pelos governos.

$\mathrm{Na}$ literatura em linguagem natural foram coletados termos relativos a games. Após a análise de cada um destes termos, utilizando a classificação inicial proposta fez-se a tentativa de traduzir cada termo encontrado para os termos criados na linguagem documentária. Essa tentativa de adequação de termo conhecido no mercado para um termo documentário é de livre iniciativa dos autores, servindo como uma proposta de classificação de jogos eletrônicos construídos para PDV com o intuito de servir como TA.

Foi realizada uma diferenciação do jogo que serve de entretenimento para 
a pessoa com deficiência visual do jogo que serve como Tecnologia Assistiva.

\section{PROPOSTA DE CLASSIFICAÇÃO DE JOGOS ELETRÔNICOS DESENVOLVIDOS COMO TECNOLOGIA ASSISTIVA PARA PESSOAS COM DEFICIÊNCIA VISUAL}

Crawford (1982) publicou a primeira obra sobre game design. Nesta foi elaborada a primeira classificação para jogos de computador, cuja questão que motivou tal pesquisa foi identificar quais eram os elementos comuns fundamentais para os jogos, percebendo então quatro fatores comuns entre eles: representação, interação, conflito e segurança. $O$ autor afirma que sua taxonomia (como ele chamou na época) não pretendia ser a correta e também afirmou não aceitar o argumento de que nenhuma que for correta poderia um dia ainda ser formulada.

Embora muito tenha se falado a respeito do uso desses em ambientes que não seja para entretenimento apenas, tais como escolas, empresas, hospitais e clínicas de saúde, muito pouco é conhecido sobre o papel de assistir diariamente as tarefas rotineiras, que encorajem hábitos saudáveis ou estilos de vida que tragam imersão e motivação aos esportes, cuidado com saúde e serviços de bem-estar. Assim esse trabalho de pesquisa tem o propósito de colaborar com a visão da construção do jogo eletrônico com elementos que sejam jogáveis pensados para assistir as atividades de pessoas com necessidades especiais, especialmente as PDV, no cuidado com os desafios diários e as características dos cuidados que devem ser contemplados.

$\mathrm{Na}$ sua construção foram considerados itens como treinamento e aprendizado, jogos de terapia e reabilitação, educação alimentar, informação sobre cuidados com a saúde, ações saudáveis, mudanças comportamentais saudáveis, estudos de interação social, estudos de avaliação cognitiva, análise da localização espacial e identificação do microambiente onde estiverem presentes estas PDV.

Algumas discussões sobre classificação são apresentadas por (TODOR, 2015) que comenta as funções dos games educativos e sugere um modelo de 
taxonomia para estes em forma de organograma. Nesta obra são apresentados os games para PDV e como afirmam Sousa e Araújo Júnior (2017), a taxonomia não é datada, ou seja, não possui uma marca temporal, não fica ultrapassada ou perde a sua utilidade, pode ser alterada a qualquer momento, com inclusão de novos elementos ou retirada de outros, por entrar em desuso ou mesmo por este ter sido contemplado em outra categoria ou classificação.

A seguir são elencadas as categorias da estrutura da classificação proposta dos jogos eletrônicos.

1. Jogos Eletrônicos

\subsection{Jogos de Ação}

1.1.1. Luta

1.1.2. Luta em 3a pessoa

1.1.3. Atirador em 3a pessoa

1.1.4. Beat'em up

1.1.5. Plataforma

1.1.6. Bola e Remo

1.1.7. Labirinto

1.1.8. Shoot'em up

1.1.9. Corrida sem fim

1.1.10. Horror

1.1.11. Ação Furtiva

1.1.12. Mundo aberto

1.1.13. Galeria de tiro

\subsection{Aventura}

1.2.1. Ficção Interativa

1.2.2. Aventura gráfica

1.2.3. Terror/Sobrevivente

\subsection{Estratégia}

1.3.1. Baseado em turnos

1.3.2. Em tempo real (RTS - Real-time strategy)

1.3.3. Defesa da Torre

1.3.4. $4 \mathrm{x}$

\subsection{Simulação}

1.4.1. Corrida de veículos

1.4.2. De vôo

1.4.3. De vida

1.4.4. De animais domésticos

1.4.5. De musica

1.4.6. Gerenciamento de Construção

\subsection{Casual}




\subsection{Quebra cabeça}

1.7. Roller Playing Game (RPG)

1.7.1. Multi User Dungeaon (MUD)

1.7.2. Massively multiplayer online role-playing game (MMORPG)

1.7.3. De ação

1.7.4. Tatil

1.7.5. Roguelike

1.7.6. Japan Roller Playing Game (JRPG)

1.8. Esporte

1.8.1. Arcadiano

1.8.2. Simulação Esportiva

1.8.3. Gerenciamento

1.8.4. Multi-esporte

1.8.5. Futebol

1.8.6. Luta como esporte

1.8.7. Gereciamento de esportes

1.9. Corrida

1.9.1. Simulador de corrida

1.9.2. Jogos de Kart

1.9.3. Veiculos de combate

1.10. Jogos Educacionais

1.10.1. Jogos educacionais individuais

1.10.2. Jogos de treinamento corporativo

1.10.3. Jogos para saúde

1.10.4. Advergame

1.10.5. Aprendizagem

1.10.6. Notícias

1.10.7. Simulação Educativa

1.10.8. Jogos persuasivos

1.10.9. Dinâmica organizacional

1.10.10. Jogos de arte

1.10.11. Militainment

1.11. Placa Eletronica

1.12. Cartas

1.13. Musica / ritmo

2. Jogos Eletrônicos para Pessoa com Deficiência Visual

\subsection{Educacional}

2.1.1. Habilidade matemática

2.1.2. Alfabetização

\subsection{RPG}

\subsection{Hobby}


2.4. Tenis

2.5. Desafio

2.6. Oráculo

2.7. Cognitivo

2.8. Memória

2.9. Motivacional

2.10. De socialização

2.11. Recreativos

2.12. De psicomotricidade

2.13. De empatia

2.14. De orientação geográfica (localização)

2.15. Outros

Ao iniciar a classificação apresentada acima a primeira inquietação dos pesquisadores foi: por que colocar, antes de qualquer classificação, a divisão entre Jogos para videntes $\mathrm{x}$ Jogos para PDV? Esta decisão levou a outras questões: todos os jogos que são desenvolvidos para um grupo devem também ser desenvolvidos para o outro? O que de fato deveria fortalecer a exigência de existir um similar para videntes e PDV?

As demandas de informação dos públicos são as mesmas, tendo apenas a característica de ser o oposto do outro ou um público apresenta uma determinada demanda e o outro apresenta outra totalmente diferente? Nesse caso, por que não colocar então a classificação sem a divisão entre jogos para videntes e jogos para PDV e no final de cada ramificação, dividir este nestes dois grupos, videntes e PDV. E sobre o jogo que não tiver o seu par, deveria ficar em branco?

\section{CONSIDERAÇÕES FINAIS}

Este estudo constatou por meio de revisão bibliográfica que as pessoas com deficiência visual não participam do principal público dos jogos eletrônicos. Propõe-se discutir a inclusão dessas por meio de maior planejamento no desenvolvimento dos jogos, justificando o uso de jogos como fonte de 
informação e recurso de apoio à apropriação da informação em outros contextos que não só o entretenimento, assim influenciando na acessibilidade à informação.

O objetivo de uma classificação não é esgotar o assunto criando um marco definitivo dos termos utilizados, mas analisar quais são esses termos e aceitar que os mesmos sejam mutáveis, adaptáveis ao longo das novas descobertas. Por esta razão a proposta deste artigo deve estar sempre aberta a atualizações.

Para diferenciar as principais características que não possibilitam a inclusão da pessoa com deficiência visual no mercado, esta pesquisa pretendeu criar uma classificação após explorar e analisar os vários gêneros de jogos, no intuito de que o mercado de jogos eletrônicos aumente sua visão para a inclusão social.

Este trabalho de pesquisa servirá para contribuir com uma classificação mais madura, mais definida, à medida que uma versão dela é experimentada, praticada pelo seu público alvo. Novos jogos eletrônicos serão desenvolvidos para a PDV e é necessário despertar o interesse nesse sentido, o que poderá levar à popularização da acessibilidade para a melhora da qualidade de vida dessas pessoas. Este artigo também pretende colaborar com a discussão e o olhar para a vulnerabilidade social que a PDV enfrenta na sociedade da informação

Por se tratar de um estudo preliminar, não pretende esgotar o assunto, e vai tomando uma forma maior e mais consistente a cada iteração, a cada surgimento de um novo elemento ou à medida que é identificada uma nova categoria, fruto da análise de um ou mais elementos que estejam intrinsicamente conectados. Por se tratar de uma linguagem documentária esta classificação pode ser aproveitada como um catálogo de jogos eletrônicos disponíveis aos usuários seja com objetivos mercadológicos ou sociais.

\section{REFERÊNCIAS}


BASTOS, K. V. S. Os desafios encontrados no acesso à informação digital por pessoas com deficiência visual. Dissertação (Mestrado em Ciência da Informação). Universidade de Brasília. Brasil: Brasília, 2017.

BERNARDO, C. G.; MORI, A.; ORLANDI, T. R. C.; DUQUE, C. G. Multimodality by Electronic Games as Assistive Technology for Visual Disabilities. In: International Conference on Technology and Innovation in Sports, Health and Wellbeing, 2016. Anais [...]. University of Trás-os-Montes e Alto Douro, Vila Real, Portugal, 2016.

BING. Página inicial da pesquisa. Disponível em: https://www.bing.com. Acesso em: 5 fev. 2018.

BRASIL. Presidência da República. Casa Civil. Decreto 5.904, de 21 de setembro de 2006. Dispõe sobre o direito da pessoa com deficiência visual de ingressar e permanecer em ambientes de uso coletivo acompanhada de cão-guia e dá outras providências. 2006. Disponível em: http://www.planalto.gov.br/ccivil_03/_Ato2004-2006/2006/Decreto/D5904.htm. Acesso em: 27 set. 2016.

BUCKLAND, M. K. A natureza da Ciência da Informação e a sua importância para a sociedade. Aula inaugural 2018 da pós-graduação em Ciência da Informação da Universidade Federal do Pará (UFPA), Brasil. Informação e Informação, Londrina, v. 23, n. 3, set./dez. 2018.

COSTA, R. Quais são os gêneros de videogames? Studio Design Zero Um. Mar, 2014. Disponível em: https://designzeroum.com.br/quais-sao-os-generosde-jogos-de-video-game/. Acesso em: 29 Dez. 2017.

CRAWFORD; C. The Art of Computer Game Design. 1982. Disponível em: https://www.digitpress.com/library/books/book art of computer game design. pdf. Acesso em: 04 jan. 2018.

CUNHA, J. H. C; ARAÚJO JUNIOR. R. H. de. Taxonomia de distorções contábeis. Encontros Bibli: revista eletrônica de biblioteconomia e ciência da informação, v. 22, n. 49, maio/ago., 2017.

CURRÁS, E. Ontologias, taxonomia e tesauro em teoria e sistemática de sistemas. Brasília: Thesaurus, Brasil, 2010. 182 p.

DAHLBERG, I. Fundamentos teóricos-conceituais da classificação. Revista Biblioteconomia, Brasília, v. 6, n. 1, jan./jun., 1978.

DAHLBERG, I. Knowledge organization: a new science? Knowledge Organization, Frankfurt, v. 33, n. 1, p. 11-19, 2006. 
DOSVOX. What is DOSVOX. NCE - Centro de Computação Eletrônica. UFRJ - Universidade Federal do Rio de Janeiro, Brasil, 2018. Disponível em: http://intervox.nce.ufrj.br/dosvox/. Acesso em: 09 jan. 2018.

FLEURY, A.; NAKANO, D.; CORDEIRO, J. H. D. O. (Coords.) Mapeamento da indústria brasileira e global jogos digitais. São Paulo: BNDES, 2014.

FURTADO, T. De nerds a ciberatletas: o crescimento exponencial do esports. Disponível em: https://oglobo.globo.com/esportes/de-nerds-ciberatletascrescimento-exponencial-do-sports-21233721. Acesso em: 23 abr. 2017.

GAME ACCESSIBILITY GUIDELINES. A straightforward reference for inclusive game design. Disponível em:

http://gameaccessibilityguidelines.com/. Acesso em: 29 dez. 2017.

GAMES FOR THE BLIND - LEAP games. Science for you Research and Development in Informatics. Atenas, Grécia. Disponível em: http://www.gamesfortheblind.org/. Acesso em: 09 jan. 2018.

\section{GEE, J. P. What Video Games Have to Teach Us About Learning and Literacy, Palgrave Macmillan: New York, 2003.}

GONÇALVES, J. Indústria de games fatura US\$ 91 bilhões em 2016. Disponível em: https://olhardigital.com.br/games-e-consoles/noticia/industriade-games-faturou-us-91-bilhoes-in-2016/64846. Acesso em: 31 Dec. 2017.

IGNÁCIO, M. M. de M; Rehabilitation of the executive functions of people with schizophrenia: proposal of a serious game contextualized in the instrumental activities of daily life. Thesis (Doctorate in Psychology). Faculty of Psychology and Educational Sciences. University of Porto. Portugal, Jan / 2016.

INTERACTION DESIGN ASSOCIATION. Interaction Awards 2014 - Blind Duck Hunt. Copenhagen Institute of Interaction Design. Copenhagen, Denmark, 2014. Acessado em 13 Out 2018. Disponível em: http://awards.ixda.org/entry/2014/blind-duck-hunt/. Acesso em: 30 mar. 2020.

HUSSAIN, Y.; RASOOL, G. A taxonomy of mobile games based on structural similarity. In: International Conference on Advancements in Computational Sciences (ICACS). Anais [...]. Lahore, Paquistão, pp. 1-6, fev/2018.

LUCCHESE, F.; RIBEIRO, B. Conceituação de jogos digitais. Universidade Estadual de Campinas: Campinas, 2009.

MATSUHARA, M.; FERNANDES, L. C. Tecnologia assistiva e tecnologia da informação e comunicação: novas perspectivas para baixa visão. E-Oftalmo Jornal Digital de Oftalmologia. v. 3, n. 2. São Paulo, Brasil, 2017. 
MEEKS, L. M.; JAIN, N. R. The Guide of Assisting Students whit Disabilities - Equal Access in Health Science and Professional Education. New York: Springer Publishing Company, USA, 2016.

MEMOR-I. Scify - Science for You. Atenas, Grécia. Disponível em: http://www.scify.gr/site/en/impact-areas-en/assistive-technologies/memorien. Acesso em: 09 jan. 2018.

MONTEIRO, S. D.; FERNANDES, R. P. M.; DECARLI, G. C.; TREVISAN, G. L. Sistemas de recuperação da informação e o conceito de relevância nos mecanismos de busca: semântica e significação. Encontros Bibli: Revista Eletrônica de Biblioteconomia e Ciência da Informação, v. 22, n. 50, p. 161-175, 2017. DOI: 10.5007/1518-2924.2017v22n50p161 Acesso em: 27 mar. 2020.

MOREIRA, W.; MORAES, I. S. O assunto "classificação" na literatura brasileira de Ciência da Informação: uma análise nos anais do ENANCIB (2003-2014). Revista Informação e Informação, Londrina, v. 24, n. 1, jan./abr. 2019.

MURIEL, D.; CRAWFORD, G. Video Games as culture. Considering the role and importance of video games in contemporary society. Routledge Advances in Sociology: Oxon, UK, 2018.

ORLANDI, T. R. C; DUQUE, C. G., MORI, A., ORLANDI, M. T. A. L. Uma nova abordagem multimodal nova à educação. Biblios, n. 70, 2018.

OTTONICAR, S. L. C; SANTOS, B. R. P dos; MORAES, I. S. Aplicabilidade da competência da informação e organização do conhecimento no processo de gestão da informação. RDBCl: Campinas, v. 15, n. 3, set./dez. 2017.

PATIKOWSKI, J.F. Jogos Inclusivos: orientações de acessibilidade para jogos digitais. 2013. Dissertação (Mestrado em Ciência da Computação). Instituto de Informática, Universidade Federal do Rio Grande do Sul, Porto Alegre, Brasil, 2013.

PIVEC, M., KEARNEY, P. Jogos para a aprendizagem e aprender com jogos. Informatica, v. 31, 2007.

ROBREDO, J.; BRÄSCHER, M. (Orgs.). Passeios pelo bosque da informação: estudos sobre a representação e organização da informação e do conhecimento - EROIC. Brasília DF: IBICT, 2010. 329 p. Disponível em: http://www.ibict.br/publicacoes/eroic.pdf. Acesso em: 15 mar. 2020.

SAKUDA, L. O.; FORTIM, I. (Orgs.). II Censo da Indústria Brasileira de Jogos Digitais. Ministério da Cultura: Brasília, 2018. Disponível em: http://www.tinyurl.com/censojogosdigitais. Acesso em: 16 fev. 2019.

SANTOS, F. Como funcionam Audiogames, jogos acessíveis para deficientes visuais. Disponível em: https://www.techtudo.com.br/dicas-e- 
Claudio Gonçalves Bernardo, Cláudio Gottschalg Duque, Tomas Roberto Cotta Orlandi, Alexandre Mori Correio

Classificação de jogos eletrônicos como tecnologia assistiva para pessoas com deficiência visual

tutoriais/noticia/2016/04/como-funcionam-audiogames-jogos-acessiveis-paradeficientes-visuais.html. Acesso em: 15 jan. 2019.

SANTOS, A. D. P.; MEDOLA, F. O.; PASCHOARELLI, L. C.; LANDIM, P. C. Tecnologia Assistiva para Pessoas com deficiência visual: uma análise da produção tecnológica no Brasil. Cadernos de Prospecção: Salvador, v. 11, n. 5, dez. 2018.

SARTORETTO, M. L.; BERSCH, R. Tecnologia Assistiva e Educação - O que é Tecnologia Assistiva? Rio Grande do Sul - Brasil, 2015. Disponível em: http://www.assistiva.com.br/tassistiva.html. Acesso em: 28 mar. 2016.

SAVI, R; ULBRICHT, V. Jogos digitais educativos: Benefícios e Desafios. 2008. Dissertação (Pós-Graduação em Engenharia e Gestão do Conhecimento). Universidade Federal de Santa Catarina, Santa Catarina, Brasil, 2008.

SCHOLAR GOOGLE. Search homepage. Disponível em: https://scholar.google.com/scholar. Acesso em: 5 fev. 2018.

SCIFY - Science For You - Atenas, Grécia, 2018. Disponível em: http://www.scify.gr/site/en/who-we-are. Acesso em: 09 jan. 2018

SEARCH YAHOO. Search homepage. Disponível em: https://search.yahoo.com. Acesso em: 25 dez. 2017.

SENS, A. L.; PEREIRA, A. T. C. Reflexões sobre o design de jogos digitais acessíveis: Caso Papa Sangre e Blindside. 2015. In: CONGRESSO NACIONAL DE HIPERMÍDIA AMBIENTES DE APRENDIZAGEM, 7., Anais [...]. Sao Luis, Maranhão, 2015.

SILVA, W. P. da. Jogos digitais adaptados para alunos com deficiência visual: estudo de habilidades cognitivas do Dosvox, 2017. Dissertação (Mestrado em Educação). Faculdade de Educação - Universidade de Brasília, Brasília, 2017.

SOUSA, R. T. B. de; ARAÚJO JÚNIOR, R. H. de. Classificação e taxonomia como instrumentos eficazes para recuperação da informação. Ciência da Informação, v. 41, n. 1, p. 148-160, Jan./abr. 2013. Disponível em: http://revista.ibict.br/ciinf/article/view/1400/1578. Acesso em: 02 nov. 2016.

SOUSA, R. T. B. de; ARAÚJO JÚNIOR, R. H de. Notas de aulas da disciplina Fundamentos da Organização da Informação. Programa de Pós-Graduação em Ciências da Informação / Faculdade de Ciência da Informação (FCl) / Universidade de Brasília (UnB). Brasília, Brasil, ago./dez., 2017.

TODOR, R. Taxonomia para jogos educativos. 2015. Dissertação (Mestrado em Design) - Pontifícia Universidade Católica do Rio de Janeiro. Rio de 
Claudio Gonçalves Bernardo, Cláudio Gottschalg Duque, Tomas Roberto Cotta Orlandi, Alexandre Mori Correio

Classificação de jogos eletrônicos como tecnologia assistiva para pessoas com deficiência visual

Janeiro, Brasil, 2015. Disponível em: www2.dbd.pucrio.br/pergamum/tesesabertas/1313582_2015_completo.pdf. Acesso em: 21

dez. 2017.

VGCHARTZ - Video Game Charts. Disponível em:

http://www.vgchartz.com/gamedb/. United Kingdom. Acesso em: 31 dez. 2017.

\title{
ELECTRONIC GAMES CLASSIFICATION AS ASSISTIVE TECHNOLOGY FOR PEOPLE WITH VISUAL DEFICIENCY
}

\begin{abstract}
Introduction: Information classification belongs to one of the essential categories of Information Science. Studies on issues related to Information Organization are fundamental, as they generate reflections on information as a thing, an object to be achieved. Objective: to propose electronic games classification as assistive technology for visually impaired person, to expand the justification for using games as a source of information and a resource to support the appropriation of information in learning contexts. Methodology: the type of research adopted was literature review, removing polysemy in names and classes of researched electronic games. Concepts about electronic games were identified in bibliographic research in the 2015-2018 quadrennium. A differentiation was made between game developed for entertainment versus game developed to be assistive technology for people with visual impairments. An organization of each style of play was created in respective groupings. Results: an electronic games classification was created for people with visual impairments. Besides presenting classification, the article draws attention to social discussion, which is one of the roles of Information Science. Conclusions: The proposal is not intended to create a label on information but facilitate the understanding of formatted information for people with visual impairments. Because it is a documentary language, it can be used as a catalog of electronic games available to user, foster dialogue about the information that can be made available through the use of these artifacts and serve as an instrument for transforming social reality.
\end{abstract}

Descriptors: Information Classification. Visually impaired person. Electronic games. Assistive technology.

\section{CLASIFICACIÓN DE JUEGOS ELECTRÓNICOS COMO TECNOLOGÍA ASISTIVA PARA PERSONAS CON DISCAPACIDAD VISUAL}

\author{
RESUMEN \\ Introducción: la clasificación de la información pertenece a una de las categorías \\ esenciales de la ciencia de la información. Los estudios sobre temas relacionados con \\ la Organización de la Información son fundamentales, ya que generan reflexiones sobre
}


Claudio Gonçalves Bernardo, Cláudio Gottschalg Duque, Tomas Roberto Cotta Orlandi, Alexandre Mori Correio

Classificação de jogos eletrônicos como tecnologia assistiva para pessoas com deficiência visual

la información como una cosa, un objeto a alcanzar. Objetivo: proponer la clasificación de los juegos electrónicos como tecnología de asistencia para la persona con discapacidad visual, ampliar la justificación para usar los juegos como fuente de información y recurso para apoyar la apropiación de la información en contextos de aprendizaje. Metodología: el tipo de investigación adoptada fue la revisión de la literatura, eliminando la polisemia en los nombres y clases de juegos electrónicos investigados. Los conceptos sobre juegos electrónicos se identificaron en la investigación bibliográfica en el cuadrienio 2015-2018. Se hizo una diferenciación entre el juego desarrollado para el entretenimiento y el juego desarrollado para ser tecnología de asistencia para personas con discapacidad visual. Se creó una organización de cada estilo de juego en las respectivas agrupaciones. Resultados: se creó una clasificación de juegos electrónicos para personas con discapacidad visual. Además de presentar la clasificación, el artículo llama la atención sobre la discusión social, que es uno de los roles de la ciencia de la información. Conclusiones: La propuesta no pretende crear una etiqueta de información, sino facilitar la comprensión de información formateada para personas con discapacidad visual. Debido a que es un lenguaje documental, se puede usar como un catálogo de juegos electrónicos disponibles para el usuario, fomentar el diálogo sobre la información que puede estar disponible a través del uso de estos artefactos y servir como un instrumento para transformar la realidad social.

Descriptores: Clasificación de la información. Persona con discapacidad visual. Juegos electrónicos. Tecnología asistiva.

Recebido em: 11/01/2020

Aceito em: 27/03/2020 